PROCEEDINGS OF THE

AMERICAN MATHEMATICAL SOCIETY

Volume 128, Number 3, Pages 923-931

S 0002-9939(99)05043-1

Article electronically published on July 28, 1999

\title{
MULTIPLICATIVE STRUCTURE OF KAUFFMAN BRACKET SKEIN MODULE QUANTIZATIONS
}

\author{
DOUG BULLOCK AND JÓZEF H. PRZYTYCKI
}

(Communicated by Ronald A. Fintushel)

\begin{abstract}
We describe, for a few small examples, the Kauffman bracket skein algebra of a surface crossed with an interval. If the surface is a punctured torus the result is a quantization of the symmetric algebra in three variables (and an algebra closely related to a cyclic quantization of $U\left(\mathfrak{s o}_{3}\right)$ ). For a torus without boundary we obtain a quantization of "the symmetric homologies" of a torus (equivalently, the coordinate ring of the $S L_{2}(\mathbb{C})$-character variety of $\mathbb{Z} \oplus \mathbb{Z}$ ). Presentations are also given for the four-punctured sphere and twice-punctured torus. We conclude with an investigation of central elements and zero divisors.
\end{abstract}

\section{INTRODUCTION}

The Kauffman bracket skein module, $\mathcal{S}_{2, \infty}(M)$, is defined as follows [4, 6]: Assume that $M$ is an oriented 3-manifold, that $\mathcal{L}_{f r}$ denotes unoriented framed links in $M$ (including the empty link), and that $R$ is any commutative ring with unit. With $A$ invertible in $R$, let $S_{2, \infty}$ be the submodule of $R \mathcal{L}_{f r}$ generated by all expressions of the form

$$
\left.\nearrow^{\prime}-A \smile-A^{-1}\right)\left(\text { and } \bigcirc+A^{2}+A^{-2} .\right.
$$

The diagrams in each relation indicate framed links that can be isotoped to identical embeddings except within the neighborhood shown, where framing is vertical. Set $\mathcal{S}_{2, \infty}(M ; R, A)=R \mathcal{L}_{f r} / S_{2, \infty}$. Notation is shortened for two special cases: $\mathcal{S}_{2, \infty}(M)=\mathcal{S}_{2, \infty}\left(M ; \mathbb{Z}\left[A^{ \pm 1}\right], A\right)$ and $\mathcal{S}(M)=\mathcal{S}_{2, \infty}(M ; \mathbb{Z},-1)$.

The following standard results about skein modules will be useful later on. Only for (1) and (6) are the proofs more than elementary exercises. These two may be found in [9] and [6].

Proposition 1. (1) (Universal coefficients property). Let $r: R \rightarrow R^{\prime}$ be a homomorphism of rings (commutative with 1), making $R^{\prime}$ into an $R$ module. The identity map on $\mathcal{L}_{f r}$ induces an isomorphism of $R^{\prime}$ (and $R$ ) modules:

$$
\mathcal{S}_{2, \infty}\left(M ; R^{\prime}, r(A)\right) \cong \mathcal{S}_{2, \infty}(M ; R, A) \otimes_{R} R^{\prime} .
$$

(2) An embedding of 3-manifolds $f: M \rightarrow N$ induces the homomorphism of skein modules $f_{*}: \mathcal{S}_{2, \infty}(M ; R, A) \rightarrow \mathcal{S}_{2, \infty}(N ; R, A)$. This leads to a functor from

Received by the editors November 17, 1997 and, in revised form, May 5, 1998.

1991 Mathematics Subject Classification. Primary 57M99.

Key words and phrases. Knot, link, 3-manifold, skein module.

The first author is supported by an NSF-DMS Postdoctoral Fellowship.

(c)1999 American Mathematical Society 
the category of 3-manifolds and embeddings to the category of $R$ modules. If $N$ is obtained from $M$ by adding 2- and 3-handles, then $f_{*}$ is an epimorphism.

(3) If $M=F \times I$ for an oriented surface $F$ and an interval $I$, then $\mathcal{S}_{2, \infty}(M ; R, A)$ is an $R$ algebra with $\emptyset$ as a unit element and $L_{1} \cdot L_{2}$ defined by placing $L_{1}$ above $L_{2}$. This multiplication depends on the product structure of $M$, so we use the notation $\mathcal{S}_{2, \infty}(F ; R, A)$. An embedding of oriented surfaces $f$ : $F \rightarrow F^{\prime}$ induces the homomorphism of skein algebras $f_{*}: \mathcal{S}_{2, \infty}(F ; R, A) \rightarrow$ $\mathcal{S}_{2, \infty}\left(F^{\prime} ; R, A\right)$. This leads to a functor from the category of surfaces and embeddings to the category of $R$ algebras.

(4) If $A=-1$, then, for any $M, \mathcal{S}_{2, \infty}(M, R,-1)$ is an $R$ algebra: $L_{1} \cdot L_{2}$ is defined to be the disjoint union of links. The algebra depends only on $\pi_{1}(M)$. In particular, if $f: M \rightarrow N$ is a homotopy equivalence, then $f_{*}: \mathcal{S}_{2, \infty}(M, R,-1) \rightarrow \mathcal{S}_{2, \infty}(N, R,-1)$ is an isomorphism of algebras.

(5) The skein module $\mathcal{S}_{2, \infty}(F ; R, A)$ has a basis consisting of links on $F$ without contractible components (but including an empty link).

(6) The skein algebra $\mathcal{S}_{2, \infty}(\odot ; R, A)$ is isomorphic to $R[x, y, z]$ where the indeterminates are the boundary curves.

Let $F_{g, n}$ denote an oriented surface of genus $g$ with $n$ boundary components. We will compute $\mathcal{S}_{2, \infty}\left(F_{g, n} ; R, A\right)$ for $(g, n) \in\{(1,0),(1,1),(1,2),(0,4)\}$. One can intuit a presentation from existing computations of the $S L_{2}(\mathbb{C})$-character ring of a free group [3] and its relationship with $\mathcal{S}_{2, \infty}\left(F_{g, n} ; \mathbb{C},-1\right)[2,8]$. Verification is then a matter of confirming relations and constructing a basis. Often we will check linear independence by specializing to a simpler module:

Lemma 1. Let $(u)$ be a principal ideal in a Noetherian ring $R$, and let $\pi: R \rightarrow$ $R /(u)$ be the natural epimorphism. Suppose $\left\{v_{i}\right\}$ is a subset of a torsion free $R$ module $S$. If $\left\{v_{i} \otimes 1\right\}$ are linearly independent in the $R /(u)$ module $S \otimes_{R} R /(u)$, then $\left\{v_{i}\right\}$ are linearly independent in $S$.

Proof. Suppose $\sum a_{i} v_{i}=0$ with some $a_{j} \neq 0$. Since $R$ is Noetherian, there is a maximal $k$ such that $u^{k}$ divides each $a_{i}$. Let $a_{i}=u^{k} a_{i}^{\prime}$. No torsion in $S$ implies $\sum a_{i}^{\prime} v_{i}=0$, so $\sum\left(a_{i}^{\prime}+(u)\right)\left(v_{i} \otimes 1\right)=0$ in $S \otimes_{R} R /(u)$. Thus each $a_{i}^{\prime} \in(u)$, contradicting maximality of $k$.

In particular, one sees that a linearly independent set of links in $\mathcal{S}(M)$ is also linearly independent in $\mathcal{S}_{2, \infty}(M)$.

\section{Skein Algebras of $F_{1,1}$ AND $F_{1,0}$}

For the rest of this paper let us agree on the shorthand nomenclature curve for a simple closed curve (up to isotopy) in a surface $F$, and for the knot in $F \times I$ created by framing it vertically. Suppose that $x_{1}$ and $x_{2}$ are curves in $F_{1,1}$ that intersect once. Applying a skein relation to the single crossing of the link $x_{1} x_{2}$ resolves it into

$$
x_{1} x_{2}=A x_{3}+A^{-1} z,
$$

where $z$ and $x_{3}$ are the unique curves that meet both $x_{1}$ and $x_{2}$ once. For a unit $u \in R$ and $a$ and $b$ in an $R$ algebra, let $[a, b]_{u}$ denote the deformed commutator $u a b-u^{-1} b a$. Also, let $\delta=A^{2}-A^{-2}$. We use $R\langle\{g\} \mid\{r\}\rangle$ to denote the free $R$ algebra in non-commuting variables $\{g\}$ modulo the ideal generated by $\{r\}$. (Often the relations $r$ will be written as equations.) 
Theorem 1. With curves $x_{i}$ as above, $\mathcal{S}_{2, \infty}\left(F_{1,1} ; R, A\right)$ is presented as

$$
R\left\langle x_{1}, x_{2}, x_{3} \mid\left[x_{i}, x_{i+1}\right]_{A}=\delta x_{i+2}\right\rangle,
$$

where $i=1,2,3$ and subscripts are interpreted modulo three.

Proof. The skein algebra is generated by curves in the surface (Proposition 1(5)), which, except for the boundary, may be identified with slopes in $\mathbb{Q} \cup\left\{\frac{1}{0}\right\}$. These in turn can be organized as the vertices in a tesselation of the upper half space model of $\mathbb{H}^{2}$ by ideal triangles - their sides are geodesics connecting slopes of curves that cross once. The product of two curves that meet once resolves, with invertible coefficients, into two other curves that meet each of the original pair in a single point, e.g. Equation (1). Hence, one can express the vertices of any triangle in terms of an adjacent one. It follows that every non-boundary parallel curve is generated by $\left\{x_{i}\right\}$. The boundary, $\partial$, is obtained from the resolution

$$
z x_{3}=A^{2} x_{1}^{2}+A^{-2} x_{2}^{2}-A^{2}-A^{-2}+\partial,
$$

where $z$ is as in Equation (1).

The commutation relations follow easily by resolving the links $x_{i} x_{j}$. Any other relation among $x_{1}, x_{2}$ and $x_{3}$ reduces, using the commutators, to an expression in $\left\{x_{1}^{i} x_{2}^{j} x_{3}^{k}\right\}$. It suffices, then, to show that this set is a basis. Since $\mathcal{S}_{2, \infty}\left(F_{1,1} ; R, A\right)$ is free, the universal coefficients property allows us to restrict to $\mathcal{S}_{2, \infty}\left(F_{1,1}\right)$. By Lemma 1, linear independence may be checked in $\mathcal{S}\left(F_{1,1}\right)$, where it follows from Proposition 1(6).

The presentation in Theorem 1 is best described as a cyclic deformation of $R\left[x_{1}, x_{2}, x_{3}\right]$. There is a cyclic deformation of $U\left(\mathfrak{s o}_{3}\right)$ (Zachos terms it the cyclically symmetric Fairle rotation $[5,10])$ given by $U_{A}\left(\mathfrak{s o}_{3}\right)=R\left\langle y_{1}, y_{2}, y_{3}\right|\left[y_{i}, y_{i+1}\right]_{A}=$ $\left.y_{i+2}\right\rangle$. This is related to the skein algebra as follows.

Corollary 1. There is a well defined map from $\mathcal{S}_{2, \infty}\left(F_{1,1} ; R, A\right)$ to $U_{A}\left(\mathfrak{s o}_{3}\right)$ sending $x_{i}$ to $\delta y_{i}$. It is injective if and only if $\delta$ is not a zero divisor and surjective if and only if $\delta$ is invertible.

This is particularly interesting if $R=\mathbb{C}$, for it says there are two families of algebras parameterized by $A$-indistinguishable at generic $A$ but having different fibers at $A= \pm 1$, where one family has $U\left(\mathfrak{s o}_{3}\right)$ and the other has $\mathbb{C}\left[x_{1}, x_{2}, x_{3}\right]$.

It has also been observed that, generically, the algebra $\mathcal{S}_{2, \infty}\left(F_{1,1} \times I ; R, A\right)$ cannot be generated by fewer than three elements. It is clear, however, that one of the generators may be eliminated if $\delta$ is invertible.

Finally, we obtain a presentation of the skein algebra of a torus by adjoining one relation to the presentation in Theorem 1.

Theorem 2. $\mathcal{S}_{2, \infty}\left(F_{1,0} ; R, A\right)$ is the quotient of $\mathcal{S}_{2, \infty}\left(F_{1,1} ; R, A\right)$ by the principle ideal $\left(A^{2} x_{1}^{2}+A^{-2} x_{2}^{2}+A^{2} x_{3}^{2}-A x_{1} x_{2} x_{3}-2 A^{2}-2 A^{-2}\right)$.

Proof. Embedding $F_{1,1}$ into $F_{1,0}$ maps $\mathcal{S}_{2, \infty}\left(F_{1,1} ; R, A\right)$ onto $\mathcal{S}_{2, \infty}\left(F_{1,0} ; R, A\right)$, forcing $\partial=-\left(A^{2}+A^{-2}\right)$. Any relation that is not a multiple of $\partial+A^{2}+A^{-2}$ would imply a non-trivial relation among the standard basis elements in $\mathcal{S}_{2, \infty}\left(F_{1,0} ; R, A\right)$. Therefore, we need only express $\partial$ in the generators $x_{1}, x_{2}, x_{3}$. This is accomplished by eliminating $z$ from Equations (1) and (2). 


\section{The SKein Algebra of $F_{0,4}$}

Paralleling Section 2, choose distinct, non-boundary parallel curves $x_{1}$ and $x_{2}$ on $F_{0,4}$ that intersect in two points. Then compute

$$
x_{1} x_{2}=A^{2} x_{3}+A^{-2} z+\text { boundary curves, }
$$

where $x_{3}$ and $z$ are the unique curves whose minimal intersection with each of $x_{1}$ and $x_{2}$ is a pair of points. The similarity with Equation (1) should call to mind the triangular tessellation of $\mathbb{H}^{2}$ from the previous section. The vertices can be matched to the non-boundary parallel curves on $F_{0,4}$ so that each side of a triangle joins curves that meet in two points. Moreover, Equation (3) holds with the variables replaced by the vertices of any adjacent pair of triangles, provided the product term is the pair of shared vertices.

We can extend $R$ to $\bar{R}$ inside $\mathcal{S}_{2, \infty}\left(F_{0,4} ; R, A\right)$ by adjoining the boundary components of $F_{0,4}$. Since these are central, $\mathcal{S}_{2, \infty}\left(F_{0,4} ; R, A\right)$ is an algebra over the commutative ring $\bar{R}$. Links in the surface with no trivial or boundary parallel components form an $\bar{R}$-basis.

Suppose that $x_{1}$ separates the boundary curves $a_{1}$ and $a_{2}$ from the boundary curves $a_{3}$ and $a_{4}$. Set $p_{1}=a_{1} a_{2}+a_{3} a_{4}$. Define $p_{2}$ and $p_{3}$ similarly. Let $q=$ $a_{1} a_{2} a_{3} a_{4}+a_{1}^{2}+a_{2}^{2}+a_{3}^{2}+a_{4}^{2}$. One may check, by direct resolution or by eliminating $z$ from a resolution of $z x_{3}$, that

$$
\begin{aligned}
A^{2} x_{1} x_{2} x_{3} & =A^{4} x_{1}^{2}+A^{-4} x_{2}^{2}+A^{4} x_{3}^{2}+A^{2} p_{1} x_{1}+A^{-2} p_{2} x_{2}+A^{2} p_{3} x_{3} \\
& +q-\left(A^{2}+A^{-2}\right)^{2} .
\end{aligned}
$$

The similarity with the relation in $\mathcal{S}_{2, \infty}\left(F_{1,0} ; R, A\right)$, along with the $\mathbb{H}^{2}$ parameterization of curves in $F_{1,0}$ and $F_{0,4}$, suggests that one can pass between their skein algebras by sending $A$ to $A^{2}$ while requiring the boundary curves to satisfy ${ }^{1}$

$$
p_{i}=0 \text { and } q+\delta^{2}=0 .
$$

In any $R$ there is at least one solution given by $a_{1}=a_{2}=a_{3}=-a_{4}=A+A^{-1}$. Let $J$ be an ideal in $\mathcal{S}_{2, \infty}\left(F_{0,4} ; R, A\right)$ generated by a solution of Equation (5).

Theorem 3. With notation as above, $\mathcal{S}_{2, \infty}\left(F_{0,4} ; R, A\right)$ is presented as

$$
\left.\bar{R}\left\langle x_{1}, x_{2}, x_{3}\right|\left[x_{i}, x_{i+1}\right]_{A^{2}}=\left(A^{4}-A^{-4}\right) x_{i+2}-\delta p_{i+2}, \text { Equation (4) }\right\rangle .
$$

Proof. The curves $x_{i}$ generate as in the proof of Theorem 1. Commutators and Equation (4) are a matter of direct computation. The relations imply that the set $\left\{x_{1}^{i} x_{2}^{j} x_{3}^{k} \mid i j k=0\right\}$ spans the module, so we need only show they are linearly independent. As before, it suffices to consider the case $(R, A)=(\mathbb{Z},-1)$. Here, formal identification of variables gives an isomorphism $\mathcal{S}\left(F_{1,0}\right) \cong \mathcal{S}\left(F_{0,4}\right) / J$. One may check that $\left\{x_{1}^{i} x_{2}^{j} x_{3}^{k} \mid i j k=0\right\}$ is a basis for $\mathcal{S}\left(F_{1,0}\right)$. In particular, the same set of monomials is linearly independent in $\mathcal{S}\left(F_{0,4}\right) / J$. Finally, Lemma 1 (used four times) proves they are linearly independent in $\mathcal{S}\left(F_{0,4}\right)$.

Corollary 2. One obtains a presentation of $\mathcal{S}_{2, \infty}\left(F_{0,4} ; R, A\right)$ over $R$ from the one in Theorem 3 by adding generators $a_{1}, \ldots, a_{4}$, and by adding commutation relations to make them central.

\footnotetext{
${ }^{1}$ This was originally motivated by the double branched cover of the torus over the sphere, with four branch points.
} 
Proof. The set $\left\{x_{1}^{i} x_{2}^{j} x_{3}^{k} a_{1}^{p} a_{2}^{q} a_{3}^{r} a_{4}^{s} \mid p q r s=0\right\}$ is seen to be a basis by working over $(R, A)=(\mathbb{Z},-1)$.

\section{The Skein ALgebra of $F_{1,2}$}

This algebra is harder to describe because there are far too many simple closed curves on the surface. However, since $F_{1,2}$ and $F_{0,4}$ are homotopy equivalent, we are able to guess and verify a presentation of $\mathcal{S}_{2, \infty}\left(F_{1,2} ; R, A\right)$.

Choose curves $x_{1}, x_{2}, y_{1}$ and $y_{2}$ on $F_{1,2}$ as indicated in Figure 1. (Edges are identified top-to-bottom and left-to-right.) Let $a$ denote the boundary curve in the center of the figure and define $\bar{R}=R[a] \subset \mathcal{S}_{2, \infty}\left(F_{1,2} ; R, A\right)$. Define curves $z_{1}$ and $z_{2}$ by the resolutions $x_{1} y_{1}=A z_{1}+A^{-1} w_{1}$, and $y_{1} x_{2}=A z_{2}+A^{-1} w_{2}$.

There is a homotopy equivalence between $F_{1,2}$ and $F_{0,4}$ given by retracting either surface to the spine in Figure 2 and then identifying it with the spine in the other surface. The induced map turns Equation (4) into a relation in $\mathcal{S}\left(F_{1,2}\right)$. By experimenting with coefficients we discovered the following relation in $\mathcal{S}_{2, \infty}\left(F_{0,4} ; R, A\right)$ :

$$
\begin{aligned}
A^{2} a z_{2} z_{1} & =A^{2} a^{2}+A^{-2} z_{2}^{2}+A^{6} z_{1}^{2}+\left(y_{1} y_{2}+A^{4} x_{1} x_{2}\right) a \\
& -\left(A^{-1} x_{1} y_{2}+A^{-1} x_{2} y_{1}\right) z_{2}-\left(A x_{2} y_{2}+A^{5} x_{1} y_{1}\right) z_{1} \\
& +x_{2} y_{1} x_{1} y_{2}+A^{6} x_{1}^{2}+A^{2} x_{2}^{2}+A^{2} y_{1}^{2}+A^{-2} y_{2}^{2}-A^{2}\left(A^{2}+A^{-2}\right)^{2} .
\end{aligned}
$$

Let $C\left(t_{1}, t_{2}, t_{3}\right)$ denote the set of cyclic commutators, $\left\{\left[t_{i}, t_{i+1}\right]_{A}=\delta t_{i+2} \mid i=\right.$ $1,2,3\}$, where subscripts are taken modulo three.

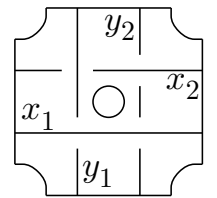

FiguRE 1
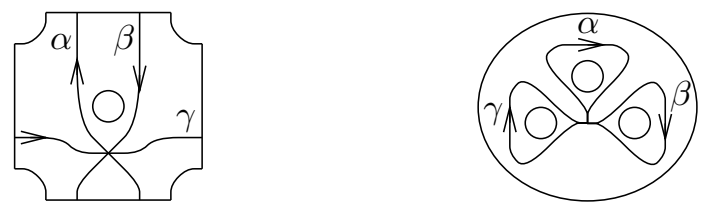

FiguRE 2

Theorem 4. $\mathcal{S}_{2, \infty}\left(F_{2,1} ; R, A\right)$ is presented ${ }^{2}$ as

$$
\begin{aligned}
\bar{R}\left\langle x_{i}, y_{i}, z_{i}\right| & {\left[x_{1}, x_{2}\right]=0,\left[y_{1}, y_{2}\right]=0,\left[z_{1}, z_{2}\right]=\delta\left(x_{2} y_{2}-x_{1} y_{1}\right), } \\
& C\left(x_{1}, y_{1}, z_{1}\right), C\left(x_{2}, y_{2}, z_{1}\right), C\left(z_{2}, y_{1}, x_{2}\right), C\left(z_{2}, y_{2}, x_{1}\right), \text { Equation (6)). }
\end{aligned}
$$

Proof. We present over $\bar{R}$ only to save the trouble of writing out trivial commutators; in the proof we work $R$-linearly. Given a handle decomposition of $F_{1,2}$, there

\footnotetext{
${ }^{2} \mathrm{~A}$ slightly different presentation was quoted in the survey article [7].
} 
is a generating set for $\mathcal{S}_{2, \infty}\left(F_{1,2} ; R, A\right)$ given in [1]. It is possible to choose the decomposition so that this set is $\left\{x_{1}, x_{2}, y_{1}, y_{2}, z_{1}, w_{2}, a\right\}$. Since $w_{2}=A y_{1} x_{2}-A^{2} z_{2}$ we can replace it with $z_{2}$.

Relations are checked by brute force. Equation (6) and commutators indicate that $\left\{a^{i} z_{2}^{j} z_{1}^{k} x_{2}^{p} y_{1}^{q} y_{2}^{r} x_{1}^{s} \mid\right.$ pqrs $\left.=0\right\}$ is a spanning set. Thus we need only show it is a basis in the case $(R, A)=(\mathbb{Z},-1)$. This follows from the homotopy equivalence in Figure 2 and the proof of Corollary 3.

\section{Central Elements And Zero Divisors}

One can prove that $\mathcal{S}_{2, \infty}(F)$ has no zero divisors and that its center is the subalgebra generated by boundary curves: All that is needed is a system for describing the standard basis so that the product of two basic elements is conveniently expressed in this language. In practice, one assigns a complexity to basis elements so that lower order terms can be ignored. In this section we will explicitly lay out these ingredients for $F_{1,0}, F_{1,1}$ and $F_{0,4}$.

5.1. Toral examples. Links in $F_{1,0}$ (with no trivial components) are denoted by points in $\mathbb{Z} \times \mathbb{Z}$, taking a point and its negative to represent the same link. The link $v=(p, q)$ is $\operatorname{gcd}(p, q)$ copies of the slope $p / q$ curve. The complexity of $(p, q)$ is $p^{2}+q^{2}$. The same conventions apply to $F_{1,1}$, treating the boundary curve as a scalar. The notation $|v \cap w|$ means the minimal geometric intersection number of $v$ and $w$ as links.

Lemma 2. Choose $v$ and $w$ in $\mathbb{Z} \times \mathbb{Z}$. The products vw and wv are expressed in the standard basis using only links in the parallelogram spanned by $\{ \pm(v \pm w)\}$. The two distinct corners occur with coefficients $A^{ \pm|v \cap w|}$.

Proof. For $v=(n, 0)$, induct on $n$. The arbitrary case transforms to this one using an element of $P S L_{2}(\mathbb{Z})$.

Theorem 5. If $A$ is not a root of unity and $R$ has no zero divisors, then the center of $\mathcal{S}_{2, \infty}\left(F_{1,0} ; R, A\right)$ is $R$ and the center of $\mathcal{S}_{2, \infty}\left(F_{1,1} ; R, A\right)$ is $R[\partial]$.

Proof. Let $\alpha=\sum r_{i} v_{i}$ with each $r_{i} \neq 0$ in $R$ and each $v_{i}$ a distinct, non-empty link in $F_{1,0}$. Choose $w$ that is not parallel to any $v_{i}$. Suppose $u$ is a maximal complexity link occurring in the resolution of $w \alpha-\alpha w$ into the standard basis. The resolution of each $r_{i}\left(w v_{i}-v_{i} w\right)$ lies in a distinct parallelogram, so $u$ must be a vertex that is contained in exactly one of them. It cannot, therefore, cancel with any other terms of the resolution. Since its coefficient is $\pm r_{i}\left(A^{\left|v_{i} \cap w\right|}-A^{-\left|v_{i} \cap w\right|}\right), \alpha$ is not central. The same proof works for $F_{1,1}$, treating $\partial$ as a scalar.

For zero divisors we will not be able to separate the resolution into distinct parallelograms. Instead we use an elementary fact about vectors in $\mathbb{R}^{n}$.

Lemma 3. Suppose $v_{1}, v_{2}, w_{1}$ and $w_{2}$ are vectors in $\mathbb{R}^{n}$ with $v_{1}+w_{1}=v_{2}+w_{2}$. If $v_{1} \neq v_{2}$, then one of $v_{1}+w_{2}$ or $v_{2}+w_{1}$ is longer than $v_{1}+w_{1}$.

Proof. Rotate so that $v_{1}+w_{1}$ lies in the first coordinate and assume that each $\left\|v_{i}+w_{j}\right\| \leq\left\|v_{1}+w_{1}\right\|$. Use both conditions to force the first coordinates of $v_{1}$ and $v_{2}$ to agree, as well as those of $w_{1}$ and $w_{2}$. Agreement in the other coordinates follows easily. 


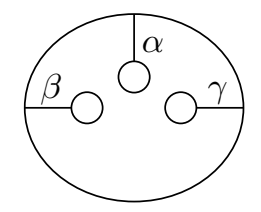

FIGURE 3

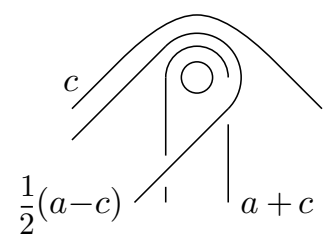

Figure 4

Theorem 6. If $R$ has no zero divisors, then $\mathcal{S}_{2, \infty}\left(F_{1,0} ; R, A\right)$ and $\mathcal{S}_{2, \infty}\left(F_{1,1} ; R, A\right)$ have no zero divisors.

Proof. Choose $\alpha=\sum r_{i} v_{i} \in \mathcal{S}_{2, \infty}\left(F_{1,0} ; R, A\right)$ with each $v_{i}$ distinct and each $r_{i} \neq 0$. Similarly, choose $\beta=\sum s_{j} w_{j}$. Suppose that $u$ has maximal complexity in the resolution of $\alpha \beta=\sum_{i, j} r_{i} s_{j}\left(v_{i} w_{j}\right)$. Reordering if necessary, we may assume that $u$ is one of $v_{1} \pm w_{1}$. If $u$ appears in the resolution of any $v_{i} w_{j}$ other than $v_{1} w_{1}$, then Lemma 3 forces a term of either $v_{1} w_{j}$ or $v_{i} w_{1}$ to have greater complexity. Thus $\alpha \beta$ expressed in the standard basis contains the non-zero term $r_{1} s_{1} A^{ \pm\left|v_{1} \cap w_{1}\right|} u$. The proof for $F_{1,1}$ is similar, noting that $R[\partial]$ has no zero divisors.

5.2. The planar example. Decorate $F_{0,4}$ with $\operatorname{arcs} \alpha, \beta$ and $\gamma$ as in Figure 3. A link in $F_{0,4}$ with no trivial components and no component parallel to an inner boundary curve defines a point $(a, b, c) \in \mathbb{N}^{3}$ as follows. Isotope it to meet $\alpha \cup \beta \cup \gamma$ minimally. The number of arcs joining $\alpha$ to $\beta$ is $a$; the number from $\beta$ to $\gamma$ is $b$; and from $\gamma$ to $\alpha, c$. A point corresponds to a link if its coordinates are congruent modulo two. There are two useful complexities for $(a, b, c): a+b+c$ and $a^{2}+b^{2}+c^{2}$. Throughout this section we assume the three inner boundary components are scalars in $\mathcal{S}_{2, \infty}\left(F_{0,4} ; R, A\right)$. Note that the extended scalars have zero divisors only if $R$ does.

Lemma 4. Using either complexity,

$$
(a, b, c)\left(a^{\prime}, b^{\prime}, c^{\prime}\right)=A^{\left(a b^{\prime}-b a^{\prime}+b c^{\prime}-c b^{\prime}+c a^{\prime}-a c^{\prime}\right) / 2}\left(a+a^{\prime}, b+b^{\prime}, c+c^{\prime}\right)+\mathcal{O},
$$

where $\mathcal{O}$ denotes lower complexity terms.

Proof. Assume for now that $a>c$. Position the link so that near $\alpha$ it looks like Figure 4, in which a number next to a strand indicates so many parallel copies. If every crossing in Figure 4 is smoothed in the vertical direction the resulting coefficient is $A^{(a-c)\left(a^{\prime}+c^{\prime}\right) / 2}$. If $c<a$ the link should be isotoped to look like a left-to-right reflection of Figure 4, but vertical resolution of every crossing yields the same coefficient. In either case, a resolution including even one horizontal smoothing allows an isotopy that removes two points of intersection with $\alpha$ (possibly by creating a scalar boundary component). 
Similarly position $(a, b, c)\left(a^{\prime}, b^{\prime}, c^{\prime}\right)$ near $\beta$ and $\gamma$. The rest of the link can be drawn with no more crossings. Therefore the complete resolution contains a term $A^{\left(a b^{\prime}-b a^{\prime}+b c^{\prime}-c b^{\prime}+c a^{\prime}-a c^{\prime}\right) / 2}\left(a+a^{\prime}, b+b^{\prime}, c+c^{\prime}\right)$. Moreover, any other link in the resolution will be some $(x, y, z)$ with $x \leq a, y \leq b, z \leq c$, and at least one inequality strict.

Theorem 7. If $R$ has no zero divisors and $A$ is not a root of unity, then the center of $\mathcal{S}_{2, \infty}\left(F_{0,4} ; R, A\right)$ is the subalgebra generated by boundary components.

Proof. We use the complexity $a+b+c$ for $(a, b, c)$. Suppose $\alpha$ is a central element. Write it as $\sum r_{i}\left(a_{i}, b_{i}, c_{i}\right)+\beta$, where each $\left(a_{i}, b_{i}, c_{i}\right)$ is distinct but all have the same complexity, and $\beta$ consists of lower complexity terms. Lemma 4 implies

$$
0=[\alpha,(2,0,0)]=\sum r_{i}\left(A^{c_{i}-b_{i}}-A^{b_{i}-c_{i}}\right)\left(a_{i}+2, b_{i}, c_{i}\right)+\mathcal{O},
$$

so $b_{i}=c_{i}$. Similar computations with $(0,2,0)$ and $(0,0,2)$ show that each $\left(a_{i}, b_{i}, c_{i}\right)$ is a power of the outer boundary curve. That means $\beta$ is central, so we can repeat the same argument for it. Continuing in this fashion, we see that every term of $\alpha$ is a scalar times a power of the outer boundary component.

Theorem 8. If $R$ has no zero divisors, then neither does $\mathcal{S}_{2, \infty}\left(F_{0,4} ; R, A\right)$.

Proof. We use the complexity $a^{2}+b^{2}+c^{2}$ for $(a, b, c)$. Choose $\alpha=\sum r_{i}\left(a_{i}, b_{i}, c_{i}\right)$ with $r_{i} \neq 0$ and each $\left(a_{i}, b_{i}, c_{i}\right)$ distinct. Similarly choose $\beta=\sum s_{j}\left(a_{j}^{\prime}, b_{j}^{\prime}, c_{j}^{\prime}\right)$. Reordering if necessary, we may assume $\left(a_{1}+a_{1}^{\prime}, b_{1}+b_{1}^{\prime}, c_{1}+c_{1}^{\prime}\right)$ is a maximal complexity link in the resolution of $\alpha \beta$. By Lemma 4, it occurs in the resolution of $r_{1} s_{1}\left(a_{1}, b_{1}, c_{1}\right)\left(a_{1}^{\prime}, b_{1}^{\prime}, c_{1}^{\prime}\right)$ with non-zero coefficient. By Lemma 3 it does not occur in any other $r_{i} s_{j}\left(a_{i}, b_{i}, c_{i}\right)\left(a_{j}^{\prime}, b_{j}^{\prime}, c_{j}^{\prime}\right)$. Hence, $\alpha \beta \neq 0$.

The central element theorems can fail at roots of unity. When $A^{2}=1$ any $\mathcal{S}_{2, \infty}(F ; R, A)$ is commutative. When $A^{4}=1, \mathcal{S}_{2, \infty}\left(F_{0,4} ; R, A\right)$ is still commutative. The toral examples are not, but the square of every curve is central.

\section{REFERENCES}

[1] D. Bullock, A finite set of generators for the Kauffman bracket skein algebra, Math. Z., to appear.

[2] D. Bullock, Rings of $S L_{2}(\mathbb{C})$-characters and the Kauffman bracket skein module, Comm. Math. Helv. 72 (1997), 521-542. CMP 98:07

[3] R. Horowiz, Characters of free groups represented in the two dimensional linear group, Comm. Pure Appl. Math. 25 (1972) 635-649. MR 47:3542

[4] J. Hoste and J. H. Przytycki, A survey of skein modules of 3-manifolds, Knots 90, de Gruyter (1992) 363-379. MR 93m:57018

[5] A. Odesskii, An analogue of the Sklyanin algebra, Funct. Anal. Appl. 20 (1986) 78-79. MR

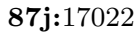

[6] J. H. Przytycki, Skein modules of 3-manifolds, Bull. Polish Acad. Science 39(1-2) (1991) 91-100. MR 94g:57011

[7] J. H. Przytycki, Introduction to algebraic topology based on knots, Proceedings of Knots 96, (S. Suzuki, ed.) World Scientific (1997) 279-297.

[8] J. H. Przytycki and A. Sikora, On skein algebras and $S L_{2}(\mathbb{C})$-character varieties, e-print: q-alg/9705011. 
[9] J. H. Przytycki and A. Sikora, Skein algebra of a group, Proc. Banach Center Mini-Semseter on Knot Theory, to appear.

[10] C. K. Zachos, Quantum deformations, Proceedings of the Argonne Workshop on Quantum Groups, (T. Curtright, D. Fairle and C. Zachos, eds.) World Scientific (1990). MR 92b:17023

Department of Mathematics, The George Washington University, Washington, DC 20052

Current address, D. Bullock: Department of Mathematics, University of Maryland, College Park, Maryland 20742

E-mail address: bullock@math.umd.edu

E-mail address: przytyck@math.gwu.edu 